\title{
Normal Half Normal Model for the Incorporation of exogenous factors on Efficiency analysis and its application
}

\author{
Shameena. H. Khan ${ }^{1 *}$, L.Mary Louis ${ }^{2}$ \\ 1* Department of Mathematics, Avinashilingam Institute for home science and higher education for women, Coimbatore, India \\ ${ }^{2}$ Faculty of Mathematics, Faculty of Engineering, Avinashilingam Institute for home science and higher education for women, \\ Coimbatore, India \\ *Corresponding Author: shaminakhan@yahoo.com,Tel.: +919629973345
}

Available online at: www.isroset.org

Received: 30/Oct/2017, Revised: 15/Nov/2017, Accepted: 24/Nov/2017, Published: 31/Dec/2017

Abstract - Present study focused on the effect of exogenous factors on the efficiency level of a firm. In this paper we have derived a model by assuming normal-half normal distribution for the error terms $v$ and $u$.Parameter estimation was done using maximum likelihood estimates. In order to assess the impact of exogenous factors on the efficiencies of a firm the model is applied to a data of 155 maize farmers. Weather, Economic conditions of farmer, Market status and damages to the crop due to bird attack are considered as exogenous factors for the study. Results showed that damages had a significant effect on the efficiency of production output. Goodness of fit test showed that our model is fitted with baseline comparison values. Null hypothesis is rejected. Computational analysis was done using SEM in Stata.

Keywords - Normal half normal, Exogenous factors, Cost efficiency, Goodness of fit, maximum likelihood estimates, Null hypothesis

\section{INTRODUCTION}

In the literature of Stochastic Frontier Analysis(SFA), in addition to the conventional inputs and outputs attention was paid to the exogenous inefficiency model as well. [10] pointed out that variables are considered exogenous in the sense that they influence the production process but are not themselves either inputs or outputs.[4] did a comparative study on exogenous and endogenous timing in social learning experiment.[6] conducted study on the students exogenous character effect on faculties and assumed $\sigma_{u}^{2}=\exp \left(z_{i}, \delta\right)$ times half normal distribution for $u_{i}$. [5] proposed a model for the estimation of inefficiency incorporated with the effect of exogenous factor on the production process. They considered half normal distribution for the inefficiency error term with mean zero and variance as $\sigma^{2}+g\left(z_{i}, \gamma\right)$ and they called it as inefficiency explanatory variable. [7,2] assumed truncated normal distribution for the inefficiency term.[9] modelled the dependence of $u(z, \delta)$ on $z$ by writing it as $u(z, \delta)=h(z, \delta) u^{*}$ where $u$ follows half normal distribution.[3] not only considered the impact of $z$ variables on the technical inefficiency part, but introduced the $z$ variables into the frontier part in a semiparametric method. Negative coefficient of the exogenous variable in the regression indicates that firms with larger values of the variables tends to have lower level of inefficiencies [8]. In this paper Normal half normal distributional assumption is considered for the inefficiency error term with exogenous effect. Parameter estimation was carried out through maximum likelihood as proposed initially by [1]. Therefore, our main aim is to contribute to this literature a new insights and to find new empirical evidences. This model is applied on a data of 155 maize farmers in the Coimbatore district of Tamil Nadu state in India

Rest of the paper is organized as follows, Section I contains the introduction and literature review, Section II we outline the model and estimation of the parameters, Section III contain point estimation of the model, Section IV describes hypothesis test, results and discussion Section $\mathrm{V}$ contain tables and figure, and Section VI concludes research work.

\section{NORMAL-HALF NORMAL EXogENOUS MODEL (NHEM)} In this formulation,

In $y_{i}=$ In $f\left(x_{i}, \beta\right)+v_{i}-u_{i}$ with $v_{i} \sim N\left(0, \sigma_{v}^{2}\right)$, they suggested the specification $\sigma_{u i}^{2}=\sigma_{u 0}^{2}+h\left(z_{i}, \gamma\right)$ with $h\left(z_{i}, \gamma\right) \geq 0$ for $u_{i} \sim N^{+}\left(0, \sigma_{u}^{2}\right)$

Rule: Mean of $u_{i}$ is a function of $\sigma_{u i}^{2}$ not zero. 
The distributional assumptions made in this paper is

1) $v_{i} \sim$ iid $N\left(0, \sigma_{v}^{2}\right)$

2) $u_{i} \sim$ iid $N\left(0, \sigma_{u}^{2}+g(z, \gamma)\right)$, here $g(z, \gamma)$ is associated with the exogenous variable.

3) $v_{i}$ and $u_{i}$ are distributed independently of each other and of the regressors

Probability density function of $\mathrm{v}$ is given by,

$f(v)=\frac{1}{\sqrt{2 \pi} \sigma_{v}} e^{\frac{-v^{2}}{2 \sigma_{v}^{2}}}$

Probability density function of $\mathrm{u}$ is given by,

$f(u)=\frac{2}{\sqrt{2 \pi} \sqrt{\sigma_{u}^{2}+g(z, \gamma)}} e^{\frac{-u^{2}}{2\left(\sigma_{u}^{2}+g(z, \gamma)\right)}}$

$f(u v)=\frac{2}{2 \pi \sigma_{v} \sqrt{\sigma_{u}^{2}+g(z, \gamma)}} e^{-\frac{v^{2}}{2 \sigma_{v}^{2}}-\frac{u^{2}}{2 \sigma_{u}^{2}+g(z, \gamma)}}$

The joint density function of $\mathrm{u}$ and $\varepsilon$ is obtained by taking the transformation $\varepsilon=v-u$

$$
\begin{aligned}
& f(u, \varepsilon)=\frac{2}{2 \pi \sigma_{v} \sqrt{\sigma_{u}^{2}+g(z, \gamma)}} e^{\frac{-(\varepsilon+u)^{2}}{2 \sigma_{v}^{2}}-\frac{u^{2}}{2\left(\sigma_{u}^{2}+g(z, \gamma)\right)}} \\
& =\frac{1}{\pi \sigma_{v} \sqrt{\sigma_{u}^{2}+g(z, \gamma)}} e^{\frac{-\left(\varepsilon^{2}+2 \varepsilon u+u^{2}\right)}{2 \sigma_{v}^{2}}-\frac{u^{2}}{2\left(\sigma_{u}^{2}+g(z, \gamma)\right)}}
\end{aligned}
$$

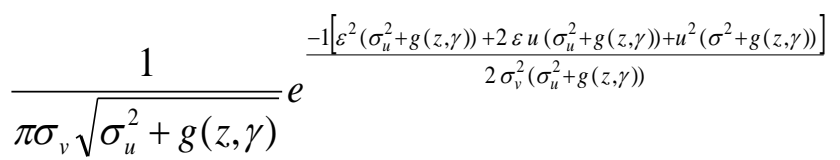

$$
\begin{aligned}
& =\frac{1}{\pi \sigma_{v} \sqrt{\sigma_{u}^{2}+g(z, \gamma)}} e^{\frac{-1\left(\sigma^{2}+g(z, \gamma)\right)}{2 \sigma_{v}^{2}\left(\sigma_{u}^{2}+g(z, \gamma)\right)}\left(u+\frac{\varepsilon\left(\sigma_{u}^{2}+g(z, \gamma)\right)}{\sigma^{2}+g(z, \gamma)}\right)^{2}} \\
& e^{\frac{-\varepsilon^{2}}{2\left(\sigma^{2}+g(z, \gamma)\right)}}
\end{aligned}
$$

The marginal density function of $\varepsilon$ is obtained by integrating $f(u, \varepsilon)$ with respect to $\mathrm{u}$

$$
f(\varepsilon)=\int_{0}^{\infty} f(u, \varepsilon) d u
$$

$$
f(\varepsilon)=\frac{1}{\pi \sigma_{v} \sqrt{\sigma_{u}^{2}+g(z, \gamma)}} e^{\left.\left.\left.\frac{-\varepsilon^{2}}{2\left(\sigma^{2}+g(z, \gamma)\right)} \int_{0}^{\infty} e^{\frac{-1}{2}\left(\frac{\sigma^{2}+g(z, \gamma)}{\sigma_{v}^{2}\left(\sigma_{u}^{2}+g(z, \gamma)\right)}\right.}\right)^{2} \frac{\varepsilon\left(\sigma_{u}^{2}+g(z, \gamma)\right)}{\sigma^{2}+g(z, \gamma)}\right]\right)} d u
$$

Let $\sqrt{\frac{\sigma^{2}+g(z, \gamma)}{\sigma_{v}^{2}\left(\sigma_{u}^{2}+g(z, \gamma)\right)}}\left[u+\frac{\varepsilon\left(\sigma_{u}^{2}+g(z, \gamma)\right)}{\sigma^{2}+g(z, \gamma)}\right]=t$

$$
\sqrt{\frac{\sigma^{2}+g(z, \gamma)}{\sigma_{v}^{2}\left(\sigma_{u}^{2}+g(z, \gamma)\right)}} d u=d t \quad, \quad d u=\sqrt{\frac{\sigma_{v}^{2}\left(\sigma_{u}^{2}+g(z, \gamma)\right)}{\sigma^{2}+g(z, \gamma)}} d t
$$

When $u=0, t=\frac{\varepsilon \sqrt{\sigma_{u}^{2}+g(z, \gamma)}}{\sigma_{v} \sqrt{\sigma^{2}+g(z, \gamma)}} ; u \rightarrow \infty, t \rightarrow \infty$

Therefore,

$$
f(\varepsilon)=\frac{1}{\pi \sigma_{v} \sqrt{\sigma_{u}^{2}+g(z, \gamma)}} e^{\frac{-\varepsilon^{2}}{2\left(\sigma^{2}+g(z, \gamma)\right)}} \int_{\frac{\varepsilon \sqrt{\sigma_{u}^{2}+g(z, \gamma)}}{\sigma_{v} \sqrt{\sigma^{2}+g(z, \gamma)}}}^{\infty} e^{\frac{-1}{2}(t)^{2}} \sqrt{\frac{\sigma_{v}^{2}\left(\sigma_{u}^{2}+g(z, \gamma)\right)}{\sigma^{2}+g(z, \gamma)}} d t
$$

$$
\begin{gathered}
\text { Since, } \sigma^{2}=\sigma_{u}^{2}+\sigma_{v}^{2} \\
f(u, \varepsilon)=\frac{1}{\pi \sigma_{v} \sqrt{\sigma_{u}^{2}+g(z, \gamma)}} e^{\frac{-1\left(\sigma^{2}+g(z, \gamma)\right)}{2 \sigma_{v}^{2}\left(\sigma_{u}^{2}+g(z, \gamma)\right)}\left[u^{2}+\frac{\varepsilon^{2}\left(\sigma_{u}^{2}+g(z, \gamma)\right)+2 \varepsilon u\left(\sigma_{u}^{2}+\right.}{\sigma^{2}+g(z, \gamma)}\right.} \\
=\frac{1}{\pi \sigma_{v} \sqrt{\sigma_{u}^{2}+g(z, \gamma)}} e^{\frac{-1\left(\sigma^{2}+g(z, \gamma)\right)}{2 \sigma_{v}^{2}\left(\sigma_{u}^{2}+g(z, \gamma)\right)}\left[\left(\frac{\varepsilon\left(\frac{\varepsilon\left(\sigma_{u}^{2}+g(z, \gamma)\right)}{\sigma^{2}+g(z, \gamma)}\right)^{2}}{\varepsilon^{2}\left(\sigma_{u}^{2}+g(z, \gamma)\right)}-\left(\frac{\varepsilon\left(\sigma_{u}^{2}+g(z, \gamma)\right)}{\sigma^{2}+g(z, \gamma)}\right)^{2}\right]\right.}
\end{gathered}
$$$$
f(u, \varepsilon)=\frac{1}{\pi \sigma_{v} \sqrt{\sigma_{u}^{2}+g(z, \gamma)}} e^{\frac{-1\left(\sigma^{2}+g(z, \gamma)\right)}{2 \sigma_{v}^{2}\left(\sigma_{u}^{2}+g(z, \gamma)\right)}\left[u^{2}+\frac{\varepsilon^{2}\left(\sigma_{u}^{2}+g(z, \gamma)\right)+2 \varepsilon u\left(\sigma_{u}^{2}+g(z, \gamma)\right)}{\sigma^{2}+g(z, \gamma)}\right]}=\frac{\sigma_{v}\left(\sqrt{\left.\sigma_{u}^{2}+g(z, \gamma)\right)}\right.}{\pi \sigma_{v} \sqrt{\sigma_{u}^{2}+g(z, \gamma)} \sqrt{\sigma^{2}+g(z, \gamma)}} e^{\frac{-\varepsilon^{2}}{2\left(\sigma^{2}+g(z, \gamma)\right)}} \int^{\frac{-1}{2}(t)^{2}} d t
$$$$
=
$$

$$
\frac{2}{\sqrt{2 \pi} \sqrt{\sigma^{2}+g(z, \gamma)}} e^{\frac{-\varepsilon^{2}}{2\left(\sigma^{2}+g(z, \gamma)\right)}} \frac{1}{\sqrt{2 \pi}} \int_{\frac{\varepsilon \sqrt{\sigma_{u}^{2}+g(z, \gamma)}}{\sigma_{v} \sqrt{\sigma^{2}+g(z, \gamma)}}}^{\infty} e^{\frac{-1}{2}(t)^{2}} d t
$$

Simplifying further, $=\frac{2}{\sqrt{\sigma^{2}+g(z, \gamma)}} \frac{1}{\sqrt{2 \pi}} e^{\frac{-\varepsilon^{2}}{2\left(\sigma^{2}+g(z, \gamma)\right)}} \Phi\left(-\frac{\varepsilon \sqrt{\sigma_{u}^{2}+g(z, \gamma)}}{\sigma_{v} \sqrt{\sigma^{2}+g(z, \gamma)}}\right)$ 
The likelihood function of the sample is the product of the density function of the individual observations, which is given as,

$$
L=\prod_{i=1}^{K} f\left(\varepsilon_{i}\right)
$$

The log likelihood equation for a sample of $\mathrm{K}$ producers is ,

$$
\begin{aligned}
& \text { In } L=K-\frac{1}{2} \operatorname{In}\left(\sigma^{2}+g(z, \gamma)\right)-\frac{1}{2} \operatorname{In} 2 \pi- \\
& \frac{1}{2} \sum_{i=1}^{k} \frac{\varepsilon^{2}}{\sigma^{2}+g(z, \gamma)}+\sum_{i=1}^{k} \operatorname{In} \Phi\left(-\frac{\varepsilon \sqrt{\sigma_{u}^{2}+g(z, \gamma)}}{\sigma_{v} \sqrt{\sigma^{2}+g(z, \gamma)}}\right)
\end{aligned}
$$

\section{Estimation of the parameters of Normal Half Normal Exogenous Model}

Using the first order conditions of the maximization of loglikelihood function parameters like $\sigma_{v}^{2}, \beta, \sigma^{2}, \sigma_{u}^{2}$ can be estimated.

$$
\begin{aligned}
& \frac{\partial \operatorname{In} L}{\partial \sigma_{v}^{2}}=\frac{1}{2 \sigma_{v}^{3}} \frac{\varepsilon \sqrt{\sigma_{u}^{2}+g(z, \gamma)}}{\sqrt{\sigma^{2}+g(z, \gamma)}} \frac{\sum_{i=1}^{k} \phi\left(-\frac{\varepsilon \sqrt{\sigma_{u}^{2}+g(z, \gamma)}}{\sigma_{v} \sqrt{\sigma^{2}+g(z, \gamma)}}\right)}{\sum_{i=1}^{k} \Phi\left(-\frac{\varepsilon \sqrt{\sigma_{u}^{2}+g(z, \gamma)}}{\sigma_{v} \sqrt{\sigma^{2}+g(z, \gamma)}}\right)(16)} \\
& \frac{\partial \operatorname{In} L}{\partial \beta}=\frac{x_{i} \sum_{i=1}^{k} y_{i}-\beta x_{i}}{\left(\sigma^{2}+g(z, \gamma)\right)}+x_{i} \frac{\sum_{i=1}^{k} \phi\left(-\frac{\left(y_{i}-\beta x\right) \sqrt{\sigma_{u}^{2}+g(z, \gamma)}}{\sum_{i=1}^{k} \sqrt{\sigma^{2}+g(z, \gamma)}}\right)}{\left(-\frac{\left(y_{i}-\beta x\right) \sqrt{\sigma_{u}^{2}+g(z, \gamma)}}{\sigma_{v} \sqrt{\sigma^{2}+g(z, \gamma)}}\right)}
\end{aligned}
$$

$$
\begin{aligned}
& \frac{\partial \operatorname{In} L}{\partial \sigma^{2}}=\frac{-1}{2\left(\sigma^{2}+g(z, \gamma)\right.}+\frac{\sum_{i-1}^{k} \varepsilon_{i}^{2}}{2\left(\sigma^{2}+g(z, \gamma)\right)^{2}}- \\
& \frac{\varepsilon_{i} \sqrt{\sigma_{u}^{2}+g(z, \gamma)}}{2 \sigma_{v}\left(\sigma^{2}+g(z, \gamma)\right)^{\frac{3}{2}}} \frac{\sum_{i=1}^{k} \phi\left(-\frac{\varepsilon_{i} \sqrt{\sigma_{u}^{2}+g(z, \gamma)}}{\sum_{i=1}^{k} \sqrt{\sigma^{2}+g(z, \gamma)}}\right)}{\left(-\frac{\varepsilon_{i} \sqrt{\sigma_{u}^{2}+g(z, \gamma)}}{\sigma_{v} \sqrt{\sigma^{2}+g(z, \gamma)}}\right)}
\end{aligned}
$$

$$
\begin{aligned}
& \frac{\partial \operatorname{In} L}{\partial \sigma_{u}^{2}}=\frac{-\varepsilon}{2 \sigma_{v} \sqrt{\left(\sigma_{u}^{2}+g(z, \gamma)\right)\left(\sigma^{2}+g(z, \gamma)\right)}} \\
& \sum_{i=1}^{k} \phi\left(-\frac{\varepsilon \sqrt{\sigma_{u}^{2}+g(z, \gamma)}}{\sigma_{v} \sqrt{\sigma^{2}+g(z, \gamma)}}\right) \\
& \sum_{i=1}^{k} \Phi\left(-\frac{\varepsilon \sqrt{\sigma_{u}^{2}+g(z, \gamma)}}{\sigma_{v} \sqrt{\sigma^{2}+g(z, \gamma)}}\right)
\end{aligned}
$$

Maximum likelihood estimates of the parameters of the model is obtained by equating right hand side of equations (16), (17), (18) and (19) to zero and solving those equations.

Theorem: The conditional distribution of $u$ given $\varepsilon$ is that of $u_{i} \curvearrowright N^{+}\left(0, \sigma_{u}^{2}+g(z, \gamma)\right)$ variable truncated at zero.

\section{Proof}

$$
\begin{aligned}
& f(u / \varepsilon)=\frac{f(u, \varepsilon)}{f(\varepsilon)} \\
& f(u, \varepsilon)=\frac{\frac{1}{\pi \sigma_{v} \sqrt{\sigma_{u}^{2}+g(z, \gamma)}} e^{\frac{-\left(\sigma^{2}+g(z, \gamma)\right)}{2 \sigma_{v}^{2}\left(\sigma_{u}^{2}+g(z, \gamma)\right)}\left(u+\frac{\varepsilon\left(\sigma_{u}^{2}+g(z, \gamma)\right)}{\sigma^{2}+g(z, \gamma)}\right)^{2} \frac{-\varepsilon^{2}}{e^{2\left(\sigma^{2}+g(z, \gamma)\right)}}}}{\frac{2}{\sqrt{\sigma^{2}+g(z, \gamma)}} \frac{1}{\sqrt{2 \pi}} e^{\frac{-\varepsilon^{2}}{2\left(\sigma^{2}+g(z, \gamma)\right)}} \Phi\left(-\frac{\varepsilon \sqrt{\sigma_{u}^{2}+g(z, \gamma)}}{\sigma_{v} \sqrt{\sigma^{2}+g(z, \gamma)}}\right)}
\end{aligned}
$$

$$
=\frac{\sqrt{2 \pi} \sqrt{\sigma^{2}+g(z, \gamma)}}{2 \pi \sigma_{v} \sqrt{\sigma_{u}^{2}+g(z, \gamma)}} \Phi\left(-\frac{\varepsilon \sqrt{\sigma_{u}^{2}+g(z, \gamma)}}{\sigma_{v} \sqrt{\sigma^{2}+g(z, \gamma)}}\right)^{-1}
$$

Let $\mu_{*}=\frac{\varepsilon\left(\sigma_{u}^{2}+g(z, \gamma)\right)}{\sigma^{2}+g(z, \gamma)}$ and

$\sigma_{*}=\frac{\sigma_{v} \sqrt{\sigma_{u}^{2}+g(z, \gamma)}}{\sqrt{\sigma^{2}+g(z, \gamma)}}$

$$
\begin{aligned}
& f(u, \varepsilon)=\frac{\sqrt{\sigma^{2}+g(z, \gamma)}}{\sqrt{2 \pi} \sigma_{v} \sqrt{\sigma_{u}^{2}+g(z, \gamma)}} \Phi\left(-\frac{\mu_{*}}{\sigma_{*}}\right)^{-1} \\
& e^{\frac{-1}{2}\left(\frac{u+\mu}{\sigma_{*}}\right)^{2}}
\end{aligned}
$$




\section{Point estimation of Normal Half Normal Exogenous Model}

As $f(u / \varepsilon)$ is distributed as $N^{+}\left(\mu_{*}, \sigma_{*}^{2}\right)$, the mean of this distribution serves as a point estimator of $u_{i}$ which is given by

$E(u / \varepsilon)=\int_{0}^{\infty} u f(u / \varepsilon) d u$
$E(u / \varepsilon)=\int_{0}^{\infty} u \frac{\sqrt{\sigma^{2}+g(z, \gamma)}}{\sqrt{2 \pi} \sigma_{v} \sqrt{\sigma_{u}^{2}+g(z, \gamma)}} \Phi\left(-\frac{\mu_{*}}{\sigma_{*}}\right)^{-1} e^{\frac{-1}{2}\left(\frac{u+\mu}{\sigma_{*}}\right)^{2}} d u$

Let $\frac{u+\mu_{*}}{\sigma_{*}}=t \quad$ where $\quad u=\sigma_{*} t-\mu_{*}$ and $d u=\sigma_{*} d t$

When $u=0, t=\frac{\mu_{*}}{\sigma_{*}} \quad$ and $u \rightarrow \infty, t \rightarrow \infty$ $E(u / \varepsilon)=\frac{\sqrt{\sigma^{2}+g(z, \gamma)}}{\sqrt{2 \pi} \sigma_{v} \sqrt{\sigma_{u}^{2}+g(z, \gamma)}} \int_{\frac{\mu_{*}}{\sigma_{*}}}^{\infty}\left(\sigma_{*} t-\mu_{*}\right) \Phi\left(-\frac{\mu_{*}}{\sigma_{*}}\right)^{-1} e^{\frac{-1}{2}\left(t_{*}\right)^{2}} \sigma_{*} d t$

$\frac{\sqrt{\sigma^{2}+g(z, \gamma) \Phi}\left(-\frac{\mu_{*}}{\sigma_{*}}\right)^{-1}}{\sqrt{2 \pi} \sigma_{v} \sqrt{\sigma_{u}^{2}+g(z, \gamma)}} \int_{\frac{\mu_{*}}{\sigma_{*}}}^{\infty} \sigma_{*}^{2} t e^{\frac{-1}{2\left(t_{*}\right)^{2}}} d t$ $-\frac{\sqrt{\sigma^{2}+g(z, \gamma) \Phi}\left(-\frac{\mu_{*}}{\sigma_{*}}\right)^{-1}}{\sqrt{2 \pi} \sigma_{v} \sqrt{\sigma_{u}^{2}+g(z, \gamma)}} \int_{\frac{\mu_{*}}{\sigma_{*}}}^{\infty} \mu_{*} \sigma_{*} e^{\frac{-1}{2\left(t_{*}\right)^{2}}} d t$

$\frac{\Phi\left(-\frac{\mu_{*}}{\sigma_{*}}\right)^{-1}}{\sqrt{2 \pi} \sigma_{v}} \sigma_{*} \int_{\frac{\mu_{*}}{\sigma_{*}}}^{\infty} t e^{\frac{-1}{2}\left(t_{*}\right)^{2}} d t-\frac{\Phi\left(-\frac{\mu_{*}}{\sigma_{*}}\right)^{-1} \mu_{*}}{\sqrt{2 \pi}} \int_{\frac{\mu_{*}}{\sigma_{*}}}^{\infty} e^{\frac{-1}{2}\left(t_{*}\right)^{2}} d t$

$\frac{\Phi\left(-\frac{\mu_{*}}{\sigma_{*}}\right)^{-1}}{\sqrt{2 \pi} \sigma_{v}} \sigma_{*} \int_{\frac{\mu_{*}}{\sigma_{*}}}^{\infty} t e^{\frac{-1}{2}\left(t_{*}\right)^{2}} d t-\frac{\Phi\left(-\frac{\mu_{*}}{\sigma_{*}}\right)^{-1} \mu_{*}}{\sqrt{2 \pi}} \int_{\frac{\mu_{*}}{\sigma_{*}}}^{\infty} e^{\frac{-1}{2}\left(t_{*}\right)^{2}} d t$
Let $s=\frac{t^{2}}{2}, d s=t d t \quad$; when $\quad t=\frac{\mu_{*}}{\sigma_{*}}, s=\frac{\left(\frac{\mu_{*}}{\sigma_{*}}\right)^{2}}{2}$

and $t \rightarrow \infty, s \rightarrow \infty$

$$
\begin{aligned}
& =\Phi\left(-\frac{\mu_{*}}{\sigma_{*}}\right)^{-1} \frac{\sigma_{*}}{\sqrt{2 \pi}} e^{\frac{\left(\mu_{*}\right)^{2}}{2}}-\Phi\left(-\frac{\mu_{*}}{\sigma_{*}}\right)^{-1} \mu_{*} \Phi\left(-\frac{\mu_{*}}{\sigma_{*}}\right) \\
& =\sigma_{*} \Phi\left(-\frac{\mu_{*}}{\sigma_{*}}\right)^{-1} \phi\left(-\frac{\mu_{*}}{\sigma_{*}}\right)-\mu_{*}
\end{aligned}
$$

Once the inefficiency has been estimated, the effect of each environmental variable on technical inefficiency can be calculated from

$$
\frac{\partial E\left(u_{i} / \varepsilon_{i}\right)}{\partial z_{i}}
$$

\section{RESULTS AND DISCUSSION}

The model was applied to a cross sectional data collected through multi stage stratified sampling on 155 maize farmers. Maximum likelihood estimation was carried out using structural equation modelling in STATA with technical efficiency as dependent variable and weather, marketing, economic conditions of the farmer and damage to the crop due to bird attack or natural phenomena as exogenous variables. Five Likert skills viz; Strongly agree -1, Agree-2, Neutral-3, Disagree-4 and Strongly disagree-5 was provided for the answers for the exogenous variables.

The inefficiency model for the incorporation of exogenous factors on $T e_{i}$ is given by, 
$T e_{i}=\delta_{0}+\delta_{1} x_{1 i}+\delta_{2} x_{2 i}+\delta_{3} x_{3 i}+\delta_{4} x_{4 i}$ where $x_{1 i}$

is the weather, $x_{2 i}$ market status, $x_{3 i}$ economic condition of farmer and $x_{4 i}$ damage to the crop.

Figure 1 shows the regression coefficient of weather, marketing, economic condition and damage as $0.046,0.11,0.002,0.59$ and constant as 1.2.

Hence the estimated exogenous inefficiency model is,

$T e_{i}=1.2+0.046 x_{1 i}+0.11 x_{2 i}+0.002 x_{3 i}+0.59 x_{4 i}$

The inefficiency model for the incorporation of exogenous factors on $T e_{i}$ is given by,

$T e_{i}=\delta_{0}+\delta_{1} x_{1 i}+\delta_{2} x_{2 i}+\delta_{3} x_{3 i}+\delta_{4} x_{4 i}$ where $x_{1 i}$

is the weather, $x_{2 i}$ market status, $x_{3 i}$ economic condition of farmer and $x_{4 i}$ damage to the crop.

Figure 1 shows the regression coefficient of weather, marketing, economic condition and damage as $0.046,0.11,0.002,0.59$ and constant as 1.2.

Hence the estimated exogenous inefficiency model is,

$T e_{i}=1.2+0.046 x_{1 i}+0.11 x_{2 i}+0.002 x_{3 i}+0.59 x_{4 i}$

Mean and variance of weather is 2.6 and 1; for marketing it is 2.1 and 1. Economic condition and damages of the crop due to bird attack is recorded as $2.4,1$ and $2.1,1$ respectively. Variance of technical error is given as 0.65. Damage due to bird attack was recorded as $41 \%\{(100-59) \%\}$. Which is quiet higher and has effected the efficiency of the farmers in the study area. Table 1 can be used to explain the significance of exogenous variables on efficiency. Since the probability values for weather, marketing and economic conditions are above $5 \%$ they are not significant to the technical efficiency of farmers whereas damages due to bird attack is significant to the efficiency of production output.

\section{Hypothesis test:}

Null Hypothesis: There is no correlation between variables. Alternate Hypothesis: There is correlation between variables. Results from Table 1 shows that there is correlation between the variables Economic conditions and weather; Economic condition and Damage are positively correlated. Hence we reject the null hypothesis. Table 2 results on goodness of fit of the model with root mean squared value less than 0.05 and base line comparison values closer to one shows that model is fitted.

\section{FigURES AND TABLES}

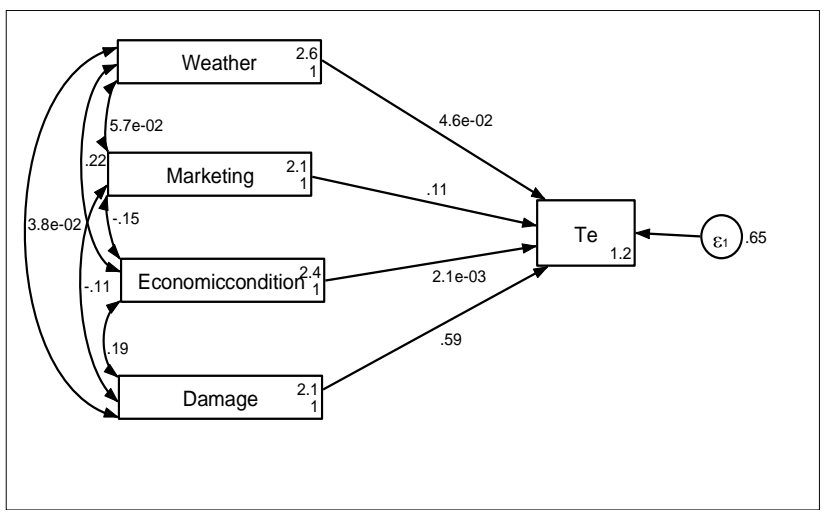

Figure. Model for the Incorporation of exogenous factors on technical efficiency.

Table 1: Maximum likelihood estimate and Hypothesis test

\begin{tabular}{|c|c|c|c|}
\hline Standardized & Std. Err. & $z$ & $P>z$ \\
\hline \multicolumn{4}{|l|}{ Structural $\mathrm{Te}<-$} \\
\hline Weather & 0.06669 & 0.68 & 0.495 \\
\hline Marketing & 0.06569 & 1.61 & 0.107 \\
\hline Economic condition & 0.06838 & 0.03 & 0.976 \\
\hline Damage & 0.04968 & 11.82 & 0.000 \\
\hline _cons & 0.32164 & 3.65 & 0.000 \\
\hline \multicolumn{4}{|l|}{ Correlation } \\
\hline \multicolumn{4}{|l|}{ Weather>- } \\
\hline Marketing & 0.08005 & 0.72 & 0.474 \\
\hline Economic condition & 0.07651 & 2.85 & 0.004 \\
\hline Damage & 0.08021 & 0.47 & 0.638 \\
\hline \multicolumn{4}{|l|}{ Marketing>- } \\
\hline Economic condition & 0.07850 & -1.91 & 0.056 \\
\hline Damage & 0.07930 & -1.4 & 0.162 \\
\hline \multicolumn{4}{|l|}{ Economic condition>- } \\
\hline Damage & 0.07750 & 2.39 & 0.017 \\
\hline Variance of $\mathrm{Te}$ & 0.05617 & & \\
\hline
\end{tabular}

Table 2: Goodness of fit

\begin{tabular}{|l|r|}
\hline Fit Statistic & \multicolumn{1}{l|}{ Fit Statistic } \\
\hline \multicolumn{2}{|l|}{ Likeli Hood ratio } \\
\hline chi2_ms(0) & 0.00 \\
\hline chi2_bs(4) & 66.12 \\
\hline p>chi2 & 0.00 \\
\hline Population error & \\
\hline RMSEA(Root mean squared error of & 0.00 \\
\hline
\end{tabular}




\begin{tabular}{|l|r|}
\hline approximation) & \\
\hline RMSE<=0.05 & \\
\hline Base line comparison & 0.999 \\
\hline CFI(Comparative fit index) & 0.956 \\
\hline TLI(Tucker-Lewis index) & \\
\hline
\end{tabular}

\section{CONCLUSION}

This paper proposes a model for the estimation of exogenous factor effect on the production efficiency and its application to a cross sectional data of 155 maize farmers. We perform SEM in Stata for the correlation and regression analysis. Technical efficiency is considered as dependent variable and exogenous factors as independent. Damage due to the bird attack had a significant impact of $41 \%$ on the efficiency level of farmers in producing the desired output. As the significance level is less than 5\% there is correlation between the variables hence we reject the null hypothesis. The paper makes contribution on the goodness of fit of the model and from the result based on RMSE and base line values we conclude that our model is fit. Focus can be given on saving the crop from the birds. In this case Government can organize training session and develop new strategies and make them reach to the farmers. This method can be extended in different fields and for other distribution also.

\section{REFERENCES}

[1] D.J.Aigner and SF. Chu, "On estimating the industry production function, American Economic Review",Vol.58, pp. 826-839,1968.

[2] S.B.Caudill ,J.M. Ford and D.M Gropper, "Frontier estimation and firm-specific inefficiency measures in the presence of heteroscedasticity", Journal of Business and Economic Statistics,Vol.13,Issue.1,pp.105 - 11,1995.

[3] Kai Sun and Subal C Kumbhakar, "Semiparametric smoothcoefficient stochastic frontier model", Economic letters, Vol.120,Issue.2,pp. 305-309,2013.

[4] Lukas Meub, Till Proeger and Hendrik Hüning, "A comparison of endogenous and exogenous timing in a social learning experiment", Centre for European Governance and Economic
Development Research, Discussion paper,Number.167,pp.126,2013.

[5] D.Reifschneider and R.Stevenson, "Systematic departures from the frontier: A framework for the analysis of firm inefficiency", International Economic Review,Vol.32,Issue.3,pp.715-23,1991.

[6] Roberto Zotti and Cristian Barra, "How students' exogenous characteristics affect faculties' inefficiency, A heteroscedastic stochastic frontier approach", Munich Personal RePEc Archive Paper No. 54011,pp.1-13,2014.

[7] L.Simar, C.A.K.Lovell and van den Eeckaut, "P.Stochastic frontiers incorporating exogenous influences on efficiency,Institut de Statistique", Universit'e Catholique de Louvain, Louvain-laNeuve, Belgium, Discussion Paper No.9403,1994.

[8] Subal C Kumbhakar, Hung-Jen wang and Alan P Horncastle, "A Practitioner's guide to stochastic frontier analysis using Stata",Cambridge University Press,ISBN:978-1-107-60946$4,2015$.

[9] Wang and Schmidt, "One-Step and Two-Step Estimation of the Effects of Exogenous Variables on Technical Efficiency Levels", Journal of Productivity Analysis, Vol.18, pp.129-144,2002.

[10] A.C Zoghbi, F.Rocha and E.Mattos, "Education production efficiency: Evidence from Brazilian universities". Economic Modelling, Vol.31, pp. 94-103,2013.

\section{AUTHORS PROFILE}

Shameena.H.Khan pursued M.Sc. in Mathematics from Govt. Brennan College affiliated to Kannur University, Kerala, India in 1999 with third rank in the university and received B.Ed in Mathematics from Keyi Sahib Training college in 2000. She is currently a Ph.D. research scholar in Avinashilingam University, Coimbatore, Tamil Nadu, India. She has presented papers in conferences and published other research papers as well. Her research is in Stochastic Frontier Analysis that consists of mathematical and statistical modelling which can be applied in various real world fields.

L.Mary Louis received her B.Sc. in Mathematics, from Holy Cross College affiliated to Madurai Kamaraj University, Madurai, Tamil Nadu, India. She also received the Ph.D. in Stochastic Frontier Analysis from Avinashilingam University, Coimbatore, Tamil Nadu, India. She is currently an Associate Professor \& Head, Avinashilingam Faculty of Engineering, Coimbatore, Tamil Nadu, India. Her research interests include Stochastic Modelling. She has published papers in reputed journals. 